\title{
Insecticide Resistance Monitoring in Culex quinquefasciatus - the Vector of Lymphatic Filariasis
}

\author{
Anju Viswan K', E Pushpalatha ${ }^{2}$, PK Srivastava ${ }^{3}$ \\ 1,2Biopesticides \& Toxicology Laboratory, Department of Zoology, University of Calicut, Malappuram, Kerala, India. \\ ${ }^{3}$ Former Joint Director and Head Vector Control, Dte. NVBDCP, Government of India, Delhi, India. \\ DOI: https://doi.org/10.24321/0019.5138.202007
}

\section{I $\quad \mathbf{N} \quad \mathbf{F} \quad \mathbf{O}$}

\section{Corresponding Author:}

Anju Viswan K, Department of Zoology, University

of Calicut, Malappuram, Kerala, India.

E-mail Id:

anjuviswan@gmail.com

Orcid Id:

https://orcid.org/0000-0001-7926-5826

How to cite this article:

Anju Viswan K, Pushpalatha E, Srivastava PK. Insecticide Resistance Monitoring in Culex quinquefasciatus - the Vector of Lymphatic Filariasis. J Commun Dis 2020; 52(1): 61-64.

Date of Submission: 2020-03-05

Date of Acceptance: 2020-03-24

\section{$\begin{array}{llllllll}\mathbf{A} & \mathbf{B} & \mathbf{S} & \mathbf{T} & \mathbf{R} & \mathbf{A} & \mathbf{C} & \mathbf{T}\end{array}$}

Objective: To assess the insecticide resistance status of Culex quinquefasciatus Say (Diptera: Culicidae) to deltamethrin, cyfluthrin and malathion in Kozhikode, Cochin, Malappuram, Thrissur and Palakkad town areas of Kerala.

Methods: Insecticide susceptibility assays were performed on adult female $C x$. quinquefasciatus mosquitoes against discriminating doses of deltamethrin $0.05 \%$, cyfluthrin $0.15 \%$ and malathion $5.0 \%$ following the procedure laid down by World Health Organisation (WHO).

Result: The data showed that $C_{x}$. quinquefasciatus is highly resistant to synthetic pyrethroids viz., deltamethrin and cyfluthrin as compared with the organophosphorus insecticide malathion.

Conclusion: The present study revealed the presence of resistance amongst the field populations of $C x$. quinquefasciatus in the study areas of five districts of Kerala. This study reflects the current status of insecticide resistance in vector of Lymphatic Filariasis in districts of Kerala which may be of immense help during the implementation of vector control strategies against $C x$. quinquefasciatus.

Keywords: $C_{x}$. quinquefasciatus, Insecticide resistance, Malathion, Deltamethrin, Cyfluthrin

\section{Introduction}

Lymphatic Filariasis (LF), commonly known as elephantiasisis caused by Wuchereria bancrofti and Brugia malayi in India but majority of the area is affected by $W$. bancrofti which is by Culex quinquefasciatus (Say), the ubiquitous mosquito breeding in large variety of water bodies. It is also known as nuisance mosquito. The disease is targeted for elimination with main strategy of preventive chemotherapy and disability alleviation. Initially, the vector control and entomological surveillance was not considered as one of the pillars of elimination strategy, however, in 2013, World health organization brought out a document of LF: Practical entomology explaining why vector control is important. ${ }^{1}$ Inpursuit to implement the appropriate vector control measures, the insecticide resistance monitoring is most important parameter so as to formulate tailor-made use of insecticide for identified area under programme. The steady development of resistance to chemical insecticides is a matter of serious concern both on economy and 
sanitation front. ${ }^{2,3}$ The insecticide resistance results in rebounding vectorial capacity and thus making it an important area of investigation in environmental health. ${ }^{4}$ The insecticide resistance monitoring has been emphasized by $\mathrm{WHO}$ as it helps in selection of appropriate insecticide and recommended dose for use under public health programme. WHO also recommends for use of standard WHO susceptibility tests for detection of insecticide resistance.

The WHO susceptibility test measures mosquito mortality to a known discriminating (diagnostic) concentration of insecticide which is set by WHO and the impregnated papers with such concentrations are supplied by WHO. The discriminating concentration conventionally corresponds to the double of the concentration at which $99.9 \%$ mortality is observed. The standard susceptibility test using the discriminating concentration, indicates that any survivors at this concentration may be considered to be resistant. However, confirmation of resistance using discriminating concentrations may not necessarily correlate with operational failure of insecticide formulations used for IRS, or for ITNs or LLINs.

The current study aims to assess the insecticidal resistance status in field populations of $C x$. quinquefasciatus of the Kozhikode, Cochin, Malappuram, Thrissur and Palakkad town areas of Kerala against malathion (5\%), cyfluthrin (0.15\%), deltamethrin (0.05\%) using WHO susceptibility kit.

\section{Materials and Methods}

The study was undertaken during the period of January 2017 to March 2018. The larvae of $C_{x}$. quinquefasciatus were collected from five different areas of Kerala State (India), viz Thrissur Municipal Corporation, Kozhikode Corporation, Cochin Municipal Corporation (Ernakulam), Palakkad
Municipality and Ponnani Municipality (Malappuram). The areas of collection are denoted as MPM-Ponnani Municipality, PKD-Palakkad Municipality, TCR-Thrissur Corporation, CLT-Kozhikode Corporation and EKM-Cochin Municipal Corporation. The larvae were brought to laboratory and transferred to a plastic tray containing water. Larval food was provided with powdered mixture of dog biscuit and yeast in the ratio 3:1. The feeding was continued until the larvae reached the fourth instar. The pupae were allowed to emerge in the mosquito cage and later the adults were used for the susceptibility tests. WHO insecticide susceptibility kit and test papers impregnated with diagnostic doses of test insecticides (Organophosphate, malathion-5\%; Pyrethroids, Cyfluthrin-0.15\%, and deltamethrin-0.05\%) were supplied by NVBDCP, Delhi. The susceptibility tests were carried out following the standard WHO procedure. ${ }^{5}$

The tests were conducted using 2-3 days old F1 generation females exposed for one hour to insecticide impregnated test and control papers. Adult response was assessed from three replicates, 20-25 females/replicate, with mortality measured 24 hours after the 1-hour exposure. Control assays included exposures of mosquitoes to papers treated only with the solvent of the insecticide in question. Knockdown was recorded after the exposure time 1 hour and final mortality was recorded after holding period of 24 hours during which time $10 \%$ sucrose solution was made availableto survivors.

\section{Result}

The percent mortality of vector mosquito $C x$. quinquefasciatus from different districts against three different insecticides viz., malathion 5\%, Cyfluthrin 0.15\% and deltamethrin $0.05 \%$ has been indicated in Table 1.

Table I.Susceptibility status of $C_{x}$. quinquefasciatus against malathion and synthetic pyrethroids in Kerala

\begin{tabular}{|c|c|c|c|c|c|c|c|}
\hline \multirow{2}{*}{$\begin{array}{c}\text { S. } \\
\text { No. }\end{array}$} & $\begin{array}{c}\text { Area of } \\
\text { collection }\end{array}$ & Insecticides used & $\begin{array}{c}\text { Total } \\
\text { mosquitoes } \\
\text { in control }\end{array}$ & $\begin{array}{c}\text { Total } \\
\text { mosquitoes } \\
\text { died in } \\
\text { control }\end{array}$ & $\begin{array}{c}\text { Total } \\
\text { mosquitoes } \\
\text { in test } \\
\text { replicates }\end{array}$ & $\begin{array}{c}\text { Total } \\
\text { mosquitoes } \\
\text { died in test }\end{array}$ & $\%$ Mortality \\
\hline \multirow{3}{*}{1.} & \multirow{3}{*}{ EKM } & Malathion 5\% & 50 & 0 & 100 & 62 & 62 \\
\cline { 3 - 8 } & & Cyfluthrin $0.15 \%$ & 50 & 0 & 100 & 24 & 24 \\
\cline { 3 - 8 } & Deltamethrin 0.05\% & 50 & 0 & 106 & 25 & 26.5 \\
\hline \multirow{3}{*}{2.} & \multirow{3}{*}{ TCR } & Malathion 5\% & 50 & 0 & 102 & 79 & 77.45 \\
\cline { 3 - 8 } & & Cyfluthrin 0.15\% & 50 & 0 & 100 & 37 & 37 \\
\cline { 3 - 8 } & Deltamethrin 0.05\% & 50 & 0 & 104 & 39 & 37.5 \\
\hline \multirow{3}{*}{3.} & \multirow{3}{*}{ PKD } & Malathion 5\% & 50 & 0 & 100 & 73 & 73 \\
\cline { 3 - 8 } & & Cyfluthrin $0.15 \%$ & 50 & 0 & 100 & 50 & 50 \\
\cline { 3 - 8 } & Deltamethrin 0.05\% & 50 & 0 & 104 & 34 & 32.69 \\
\hline
\end{tabular}




\begin{tabular}{|c|c|c|c|c|c|c|c|}
\hline & & Malathion 5\% & 50 & 0 & 100 & 78 & 78 \\
\cline { 3 - 7 } 4. & \multirow{3}{*}{ CLT } & Cyfluthrin 0.15\% & 50 & 0 & 100 & 34 & 34 \\
\cline { 3 - 8 } & & Deltamethrin 0.05\% & 50 & 0 & 100 & 28 & 28 \\
\hline \multirow{3}{*}{5.} & Malathion 5\% & 50 & 0 & 100 & 84 & 84 \\
\cline { 3 - 8 } & \multirow{2}{*}{ MPM } & Cyfluthrin 0.15\% & 50 & 0 & 100 & 46 & 46 \\
\cline { 3 - 8 } & & Deltamethrin 0.05\% & 50 & 0 & 108 & 41 & 37.96 \\
\hline
\end{tabular}

The mortality was comparatively less in EKM population than in other districts; however, in none of the places, the mortality is above $98 \%$ indicating that the vector of LF has developed resistance.

\section{Discussion}

According to WHO, mortalities below $90 \%$ represents high resistance, where as mortalities ranging from 90 to $98 \%$ represents moderate degrees of resistance and mortality above $98 \%$ represents susceptible.

The mortality of $62 \%-84 \%$ was observed against malathion, with the lowest mortality of $62 \%$ among Cochin Municipal Corporation (EKM), where as the highest mortality of $84 \%$ was observed in Mosquitoes of Ponnani Municipality (MPM). The mortality of 78,73 and 77.45 respectively was observed in Kozhikode Corporation (CLT), Palakkad Municipality (PKD) and Thrissur Corporation (TCR) mosquito population. The test also revealed high resistance against synthetic pyrethroids, as the mortality ranged between 24 to $50 \%$. The mortality of $24 \%$ against cyfluthrin was observed in Cochin Municipal Corporation, Ernakulam (EKM) followed by $34 \%$ in CLT, 37\% in TCR, 46\% in MPM and $50 \%$ in PKD. Resistance against deltamethrin was also observed, as the lowest mortality of $26.5 \%$ in EKM and the highest mortality of $37.96 \%$ in MPM were recorded. In all the three insecticides tested, $C x$. quinquefasciatus collected from Ponnani Municipality (MPM) showed the least resistance while the highest resistance was observed in individuals collected from Cochin Municipal Corporation (EKM).

The present study reveals the status of resistance against synthetic pyrethroids namely deltamethrin and cyfluthrin in field populations of $C x$. quinquefasciatus. Similar results encasing pyrethroid resistance in $C x$. quinquefasciatus have been reported world wide. ${ }^{6-10}$ Resistance to pyrethroids in Cx. quinquefasciatus have also been reported in French West Indies and West Africa. ${ }^{10}$ Synthetic pyrethroids especially deltamethrin is used in long lasting insecticidal nets (LLINs) which is one of the main strategies for malaria elimination but with the resistance, it may not be effective against vector of LF which is also known as nuisance mosquito. Low efficacy of pyrethroid impregnated bed nets against $C x$. quinquefasciatus have already been reported from various areas ${ }^{11,12}$ and this could be a major challenge of vector/ mosquito control programme in future. $C x$. quinquefasciatus being anthropophilic and indoor resting mosquito is likely to get exposed more to the house hold use of pesticides, fumigants, mosquito repellents which include synthetic pyrethroids and may be a contributing factor in precipitation of resistance. Besides, indiscriminate use of synthetic pyrethroids in agriculture practices may also facilitate in development of resistance among $C x$. quinquefasciatus.

\section{Conclusion}

Based on the current status of resistance the authors suggest that more studies on Insecticide Resistance Monitoring (IRM) are required and it is also suggested that judicious use of approved insecticides with recommended dose may be advocated by the health programme managers in such a manner that community is made aware and cautious too before using of insecticidal spray either as adulticide or larvicide.

\section{Acknowledgement}

The financial support provided by UGC BSR is highly acknowledged for carrying out the study. The technical support provided by NVBDCP Delhi and also for providing the WHO insecticide susceptibility kit and impregnated papers is gratefully acknowledged, without which it was difficult to complete the study. The authors are thankful to Department of Zoology, University of Calicut for the continuous support during the course of the study.

\section{Conflict of Interest: None}

\section{References}

1. Georghiou GP, Taylor CE. Genetic and Biological Influences in the Evolution of Insecticide Resistance. Journal of Economic Entomology 1977; 70(3): 319-323.

2. Hemingway J, Hawkes NJ, McCarroll L et al. The molecular basis of insecticide resistance in mosquitoes. Insect Biochemistry and Molecular Biology 2004; 34(7): 653-665.

3. Parimi S, Meinke LJ, Nowatzki TM et al.Toxicity of insecticide-bait mixtures to insecticide resistant and susceptible western corn root worms (Coleoptera:Chrysomelidae). Crop Protection 2003; 22(5): 781-786.

4. World Health Organization/ Department of 
Communicable Disease Prevention Control and Eradication. Techniques to detect insecticide resistance mechanisms. Field and laboratory manual. (DrM.Zaim/ WHOPES,Ed.). World Health Organization, Geneva. 1998.

5. World Health Organization. LymphaticFilariasis: PracticalEntomology-Ahand book for National Elimination Programmes. 2013.

6. Delannay C, Goindin D, Kellaou K et al. Multiple insecticide resistance in Culex quinquefasciatus populations from Guadeloupe (FrenchWestIndies) and associated mechanisms. PLoSONE 2018; 13(6): e0199615.

7. Chandre F, Darriet F, Darder M et al. Pyrethroid resistance in Culex quinquefasciatus from West Africa. Med Vet Entomol 1998; 12(4): 359-366.

8. Skovmand O, Sanogo E. Resistance of Culex quinquefasciatus to selected chemical and biological pesticides. Med Res Arch 2018; 6(4).

9. Yadouléton A, Badirou K, Agbanrin R et al. Insecticide resistance status in Culex quinquefasciatus in Benin. Parasite Vectors 2015; 8(1): 17.

10. Rai $P$, Bharati $M$, Subba $A$. Insecticide resistance mapping in the vector of lymphatic filariasis, Culex quinquefasciatus Say from northern region of West Bengal, India. PLoSONE2019; 14(5): e0217706.

11. Guillet $P, N^{\prime}$ guessan $R$, Darriet $F$ et al. Combined pyrethroid and carbamate'two-in-one'treated mosquito nets: field efficacy against pyrethroid- resistant Anopheles gambiae and Culex quinquefasciatus. Med Vet Entomol 2015; 15(1): 105-112.

12. Kudom AA, Mensah BA, FroeschlG et al. DDT and pyrethroid resistance status and laboratory evaluation of bio-efficacy of long lasting insecticide treated nets against Culex quinquefasciatus and Culex decens in Ghana. Acta Trop 2015; 150: 122-130. 